\title{
EARTHQUAKE SIGNATURE DETECTION USING CUBESAT TECHNOLOGY
}

\author{
Shivang Nayyar*1, Sandeep Kumar Rana ${ }^{2}$, Vikas Suhag ${ }^{3}$ \\ $* 14237$ Dronacharya College of Engineering, ${ }^{2} 14231$ Dronacharya College of Engineering, ${ }^{3} 14247$ Dronacharya \\ College of Engineering
}

*Corresponding Author: -

\begin{abstract}
: -
Cubesats play a vital role in development of new micro components being used in various micro/nano satellites. Cubesats have an effective small design and have the ability to get modified easily. Quakesat, a satellite being deployed by Space Systems Development Laboratory (SSDL) is used to study, detect, record, and downlink extremely low frequency (ELF) magnetic signals, which are used to predict earthquake activity. Quakesat is a live example of cubesat technology, uses non space application components and provides a low-cost alternative to launch space missions and support nano satellite infrastructure in future.
\end{abstract}

Keywords: Cubesat, Quakesat, ELF, SSDL, Ritcher Scale 


\section{INTRODUCTION}

When earthquakes of Ritcher scale magnitude of 6 or larger develop, the layers below earth's crust begin to fracture / move as stress builds along. The fracturing of the bedrocks creates the ELF magnetic waves. These signals / signatures radiate from the earthquake epicenter, through the earth $(5-80 \mathrm{Km})$, through the atmosphere to the ionosphere $(100-200$ $\mathrm{Km})$ and are propagated to the earths satellite altitude $(600-900 \mathrm{Km})$. The determination of this ground-based ELF signals is of considerable interest at the altitude of a satellite. A Russian satellite, Cosmos 1804 detected $140 \mathrm{~Hz}$ and $450 \mathrm{~Hz}$ signals just after a M6+ earthquake in Armenia in 1989 every time the satellite passed within +/- 6 degrees. of Lat. And long. of the earthquake.

Therefore, the Quakesat mission objectives are to detect, record and downlink the ELF magnetic data to verify the results from there prior experiments. Investigating the ELF signals can ultimately lead to the prediction of earthquakes of magnitude 6.0 or more. Quakesat will help to determine if the cubesat paradigm is cost effective platform for conducting significant scientific research. Quakesat is designed for a nominal one year long mission life and are deployed in a sun synchronous, low earth orbit (LEO) at an altitude of $65 \mathrm{Km}$.

ELF waves refract in the Ionosphere and propagate into space along the earth's magnetic field lines. Quakesat will use permanent magnets for passive altitude control to orient the quakesat close to the magnetic field lines. A deployable payload boom contains the ELF magnetometer which collects raw data. This boom is necessary to shield the ELF sensor from any internal magnetic disturbances generated by the quakesat electronics.

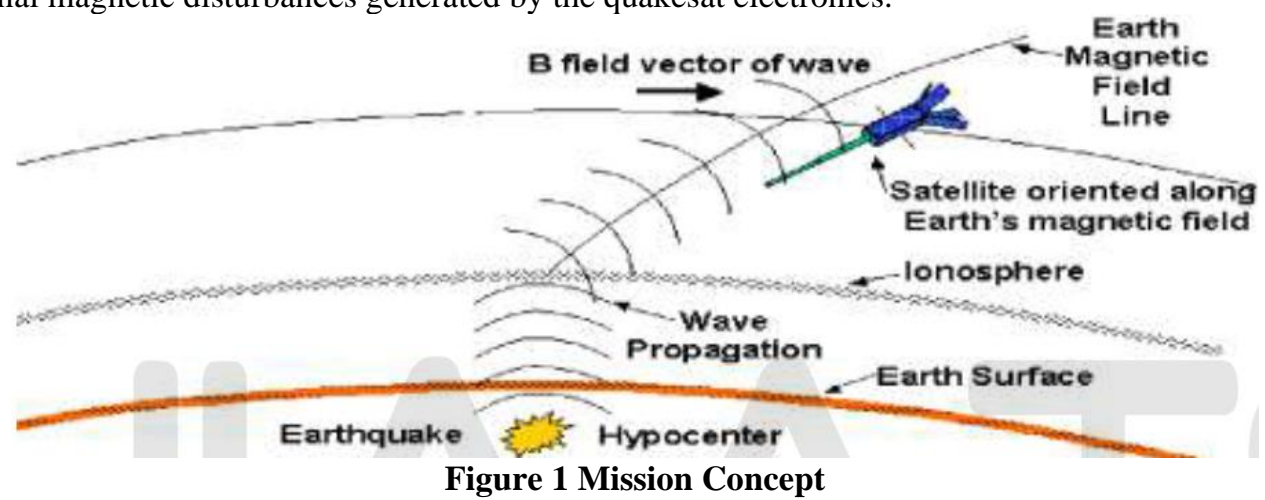

\section{PAYLOAD DESCRIPTION}

The strategy used to detect these magnetic fields relies on the use of very sensitive AC magnetometer. The ELF sensor is a single axis search coil magnetometer with multiple frequency bands. The magnetometer is $0.3 \mathrm{~m}$ long and $0.02 \mathrm{~m}$ in diameter. There is no on-board processing of the mission data other than multiple, analog low pass, high pass and band pass filters. Figure 4 shows the architecture of Quakesat. The payload was designed for simplicity but was modified to select specific frequency bands and only power those bands to keep the overall power usage to a minimum. The quakesat frequency bands for the ELF payload are: Band 1: 0.5 to $10 \mathrm{~Hz}$ narrow band magnetic; Band 2: $10 \mathrm{~Hz}$ to $150 \mathrm{~Hz}$ wide band magnetic; Band 3: $10 \mathrm{~Hz}$ to $1000 \mathrm{~Hz}$ wide band magnetic; Band 4: 130-150 Hz narrow band magnetic; Band 5: 130$150 \mathrm{~Hz}$ narrow band electric field. All the bands are sampled at 2-3x their frequency, except the 130-150 $\mathrm{Hz}$ band, which is sampled at $1 / \mathrm{sec}$. During data gathering event, one of the other wideband or narrow band filters is also commanded on, and the sample rate is adjusted accordingly.

\section{STRUCTURE}

The quakesat is a design of cubesat design itself and has a length equal to three cubesats and weighs $3 \mathrm{~kg}$. The magnetometer is fixed inside the structure after being attached to the end of the boom. Quakesat also contains an inner rack that slides inside the outer structure. The flight apparatus and circuits are fastened to the inner rack and arranged so as to fit the components. The spacecraft is symmetric when the solar arrays are fully deployed, the product of inertia is zero and center of mass only shifts along the axis.

\section{COMMAND AND DATA HANDLING}

Command and data handling of quakesat is designed to operate with timed commands to synchronize data gathering with historically active earthquake prone areas. To sample the data world is mapped into grid of 5 degree or 10-degree squares of latitude and longitude. This resolution was derived to allow sensor to be properly calibrated. The position of quakesat is determined by NORAD Two-line Element Sets and a ground-based propagator. The determination of quakesat orbital position will be calculated on the ground. The ELF data will then be down linked through one or more ground stations on the earth. It will also allow changes in sample rates of the pay load and direct on/off commanding of the payload. A capable CPU is selected to satisfy the requirements. The Prometheus processor is selected based on its wide operational temperature range.

\section{COMMUNICATION}

The major components used for communication are Tekk T net mini 9600 Baud, half duplex crystal-controlled radio and a modem. The Tekk radio is one of the smallest 9600 baud data packet radios. The communication system uses UHF frequency for both transmission and reception. Quakesat has four antennas that are quarter wavelength long about $70 \mathrm{~mm}$ and $6.5 \mathrm{~mm}$ wide. Each antenna is mounted on four corners of the satellite at 45 degrees. 


\section{ELECTRICAL POWER SYSTEM}

The electrical power system includes three subsystems: a power source, storage and a regulator. Power source consists twelve solar panels: four are body mounted and four are deployable wings. The panels are programmed keeping in mind the direction of the sun. The deployable wings are canted at an angle of 150 degrees. The power levels expected are 2.8 $\mathrm{W}$ to $12.6 \mathrm{~W}$ over the clock. Every solar panel has dimensions of $330 * 86 \mathrm{~mm}$ and produces voltage accordingly and store them in high efficiency Li+ batteries. Each charge controller limits the current and maximum charging current to 3 A. Quakesat uses a passive thermal controller and the temperature excursion is expected to be between 27-to--38-degree celcius.

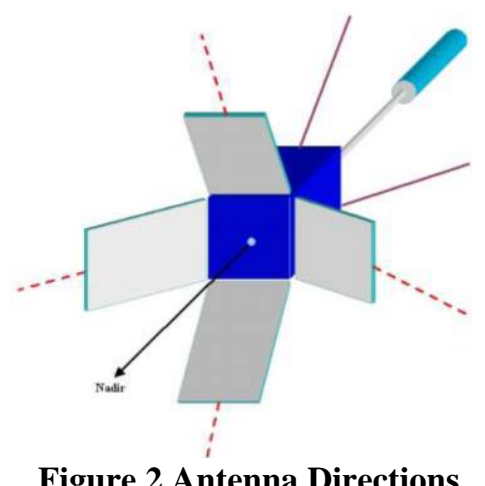

Figure 2 Antenna Directions

\section{GROUND STATION OPERATION}

Once quakesat is launched and the solar panels are deployed, the satellite is commanded through the quakesat ground station. The satellite will be given a satellite ID number for tracking purpose. Pre-determined areas of interest will be compared against the satellite track and appropriate payload on-off times will be computed using the world map. The ground station will determine which frequency gives the best possible data and then generates a file of channel on- off times. When the clock reaches the proper time, individual payload power switches will be toggled as the satellite crosses a particular area. The files are stored in the ground station server and based on the calculation done by applying various algorithms the earthquake can be detected. Based on a pre-coordinated schedule, Quakesat will download the data files with ID. Subsequently it will be transmitted using FTP to quake finder headquarters.

\section{CONCLUSION}

We see in this paper that the Quakesat is an effective small satellite that may be modified to accommodate larger academic and even commercial missions. Quakesat is being launched at the lowest of prices and hence should prove that Cubesat class of satellites provide an effective and cost alternative to larger space missions. This paper also describes the use of ELF. Quakesats mission goal is to monitor that ELF signatures can also be detected from LEO and ground stations data. Periodically the report can be generated of earthquakes of magnitude 6 and above around the world. This analysis shows if ELF signals can be detected by a satellite before and after large earthquakes.

\section{REFRENCES}

[1].A.C. Fraser Smith, et. al., "Low-frequency magnetic field measurements near the epicenter of the Ms 7.1 Loma Prieta earthquake", Geophysical Research Letters, Vol. 17, No. 9, pages 1465-1468, August 1990.

[2].O.N. Serebryakova, et. al., "Electromagnetic ELF radiation from earthquake regions as observed by low-altitude satellites", Geophysical Research Letters, Vol. 19, No. 2, pages 91- 94, January 24, 1992.

[3].M. Parrot, "Statistical study of ELF/VLF emissions recorded by a low-altitude satellite during seismic events", Journal of Geophysical Research, Vol. 99, No. A12, pages 23,339-23,347, December 1, 1994.

[4].Dallas Semiconductor, MAX1873 Li+ Charge Controller Kit, July1 2001, http://www.maximic.com/quick_view2.cfm/qv_pk/3105.

[5].Power-One Series Single Output Power Regulator, June 2002, http://www.power-one.com/.

[6].Wertz, J.R., and Larson, W.J., Space Mission Analysis and Design, 3 Ed., Microcosm Press, El Segundo, CA, 1999.

[7].Menges, B.M., Guadiamos, C.A., and Lewis, E.K., "Dynamic Modeling of Micro-Satellite Spartnik's Attitude", San Jose State University, http://www.engr.sjsu.edu/spartnik/adac.html.

[8].White, J., "Microsat Motion, Stabilization, and Telemetry", AMSAT-NA, September 1990, http://coloradosatellite.com/Papers/Motion.html.

[9].Kim, K., "Analysis of Hysteresis for Attitude Control of a Microsatellite", San Jose State University, http://www.engr.sjsu.edu/spartnik/adac.html.

[10]. Swartout, M., "Attitude Determination and Control, SapphireNotes", http://students.cec.wustl.edu/ sapphire/design/log/sec8adc/8.1.html. 